\title{
Circuit Theory for Full Counting Statistics in Multiterminal Circuits
}

\author{
Yu. V. Nazarov and D. A. Bagrets \\ Department of Applied Physics and Delft Institute of Microelectronics and Submicrontechnology, \\ Delft University of Technology, Lorentzweg 1, 2628 CJ Delft, The Netherlands
}

(Received 12 December 2001; published 25 April 2002)

\begin{abstract}
We propose a theory that treats the current, noise, and, generally, the full current statistics of electron transfer in a mesoscopic system in a unified, simple, and efficient way. The theory appears to be a circuit theory of $2 \times 2$ matrices associated with Keldysh Green functions. We illustrate the theory by considering the big fluctuations of currents in various three-terminal circuits.
\end{abstract}

DOI: $10.1103 /$ PhysRevLett.88.196801

The field of quantum noise in mesoscopic systems has exploded during the last decade, with most achievements being summarized in a recent review article [1]. Measurement of fractional charge in quantum Hall regime [2], noise measurements in atomic-size junctions [3], and superconductors [4] are milestones of the field and demonstrate the importance of quantum noise as a unique tool to study electron correlations and entanglements of different kinds. A very important step has been made in [5] where an elegant theory of full counting statistics (FCS) has been presented. This theory encompasses not only noise, but all higher momenta of the charge transfer.

Starting from the pioneering work of Büttiker [6], special attention has been paid to noise and statistics of electron transfer in multiterminal circuits. The correlations of currents flowing to different terminals reveal Fermi statistics of electrons. These cross correlations have been recently observed [7]. Although the noise correlations for several relevant layouts have been understood [1], the evaluation of FCS still encountered difficulties. For instance, an attempt to build up FCS with the "minimal correlation approach" [8] has led to contradictions [9]. This is unfortunate, since higher-order current correlations supply information about higher-order electron correlations and multiparticle interference. This information is of fundamental importance and can be hardly obtained by any other means.

In this Letter, we present a calculation scheme that allows for easy evaluation of FCS in a multiterminal mesoscopic system. It is of great intellectual enjoyment that this scheme is a simple and a universal one. In fact, it is hardly more complicated than a conventional circuit theory of electric transport and is based on a slight extension of Kirchoff rules to $2 \times 2$ matrix structures.

We start by introducing current operators $\hat{I}_{i}$, each being associated with the current to a certain terminal $i$. Extending the method of [10] we introduce a Keldysh-type Green function defined by

$\left(i \frac{\partial}{\partial t}-\hat{H}-\frac{1}{2} \bar{\tau}_{3} \sum_{i} \chi_{i}(t) \hat{I}_{i}\right) \otimes \check{G}\left(t, t^{\prime}\right)=\delta\left(1-1^{\prime}\right)$.
PACS numbers: 73.23. $-\mathrm{b}, 05.40 .-\mathrm{a}, 72.70 .+\mathrm{m}, 74.40 .+\mathrm{k}$

Here we follow notations of a comprehensive review [11], $\chi_{i}$ are time-dependent parameters, $\bar{\tau}_{3}$ is a $2 \times 2$ matrix in Keldysh space, and $\hat{H}$ is the one-particle Hamiltonian that incorporates all information about the system layout, including boundaries, defects, and all kinds of elastic scattering. We use "hat," "bar," and "check" to denote operators in coordinate space, matrices in Keldysh space, and operators in direct product of these spaces, respectively. Equation (1) defines the Green function unambiguously provided boundary conditions are satisfied: $\breve{G}\left(t, t^{\prime}\right) \equiv$ $\bar{G}\left(x, x^{\prime} ; t, t^{\prime}\right)$ approaches the common equilibrium Keldysh Green functions $\breve{G}_{i}^{(0)}\left(t-t^{\prime}\right)$ provided $x, x^{\prime}$ are sufficiently far in the terminal $i$. These $\breve{G}_{i}^{(0)}\left(t-t^{\prime}\right)$ incorporate information about the state of the terminals: their voltages $V_{i}$ and temperatures $T_{i}$.

One can easily see by traditional diagrammatic methods [11] that the expansion of $\breve{G}$ in $\chi_{i}(t)$ generates all possible diagrams for higher-order correlators of $\hat{I}_{i}(t)$ and thereby incorporates all the information about statistics of charge transfer. If we limit our attention to the low-frequency limit of current correlations, we can keep time-independent $\chi_{i}$. In this case, the Green functions are functions of time difference only and Eq. (1) separates in energy representation. It is convenient to introduce the following $\chi_{i^{-}}$ dependent action defined as a sum of closed diagrams:

$$
\frac{\partial S}{\partial \chi_{i}}=-i t_{0} \int \frac{d \varepsilon}{2 \pi} \operatorname{Tr}\left(\bar{\tau}_{3} \hat{I}_{i} \breve{G}(\varepsilon)\right) .
$$

This allows us to express the probability for $N_{i}$ electrons to be transferred to the terminal $i$ during time interval $t_{0}$,

$$
P\left(\left\{N_{i}\right\}\right)=\int_{-\pi}^{\pi} \prod_{i} \frac{d \chi_{i}}{2 \pi} e^{-S\left(\left\{\chi_{i}\right\}\right)-i \sum_{i} N_{i} \chi_{i}} .
$$

(Higher-order) derivatives of $S$ with respect to $\chi_{i}$ give (higher-order) moments of $P\left(\left\{N_{i}\right\}\right)$. First derivatives yield average currents to terminals; second derivatives correspond to the noises and noise correlations.

Using special properties of current operators, $\chi$ dependent terms in Eq. (1) can be gauged away $[10,12]$. The $\chi$ dependence of $G$ is thereby transferred to the boundary conditions: the gauged Green function far in each 
terminal shall approach $\breve{G}_{i}(\epsilon)$ defined as

$$
\check{G}_{i}(\epsilon)=\exp \left(i \chi_{i} \bar{\tau}_{3} / 2\right) \check{G}_{i}^{(0)}(\epsilon) \exp \left(-i \chi_{i} \bar{\tau}_{3} / 2\right) .
$$

The precise form of the functions $\check{G}_{i}^{(0)}(\epsilon)$ is defined below in the text [see Eq. (8)].

In the present form, Eq. (1) with relations (4),(2) solves the problem of determination of the FCS for any arbitrary system layout: one just has to find the exact quantummechanical solution of a Green function problem. This is hardly constructive, and we proceed further by deriving a simplified semiclassical approach. First, we note that even in its exact quantum-mechanical form Eq. (1) possesses an important property. We consider the quantity defined similar to the standard definition of current density, $\bar{j}^{\alpha}(x, \epsilon) \equiv \lim _{x \rightarrow x^{\prime}}\left(\nabla^{\prime \alpha}-\nabla^{\alpha}\right) \bar{G}\left(x, x^{\prime} ; \epsilon\right) / m$. By virtue of Eq. (1) this quantity conserves so that

$$
\partial \bar{j}^{\alpha}(x, \epsilon) / \partial x^{\alpha}=0 .
$$

This looks like the conservation of particles at a given energy. However, this relation contains more information since it is a conservation law for a $2 \times 2$ matrix current.

Next, we construct a theory which makes use of this conservation law. We concentrate on the semiclassical Green function in coinciding points, $\bar{G}(x, \epsilon) \equiv i \bar{G}\left(x, x^{\prime} ; \epsilon\right) / \pi \nu$ $[11,13,14]$. It satisfies the normalization condition $\bar{G}^{2}=$ $\overline{1}$. We relate the "current density" $\bar{j}$ to gradients and/or changes of $\bar{G}(x)$, very much like the electric current density is related to the voltage in circuit theory of electric conductance. Following the approach of the circuit theory, we separate a mesoscopic layout into elements: nodes and connectors, so that $\bar{G}(x)$ is constant across the nodes and drops across the connectors (see Fig. 1). This separation of actual layout is rather heuristic, similar to the separation of an electric conductor of a complicated geometry onto nodes and circuit theory elements. The bigger the number and the finer the mesh of the nodes and connectors, the better the circuit theory approximates the actual layout. The nodes are similar to the terminals; the difference is that $\bar{G}$ is fixed in the terminals and yet to be determined in the nodes. The $\bar{G}$ in nodes are determined from Kirchoff rules reflecting the conservation law (5): the sum of the matrix currents from the node over all connectors should equal zero at each energy. For this, we should be able to express

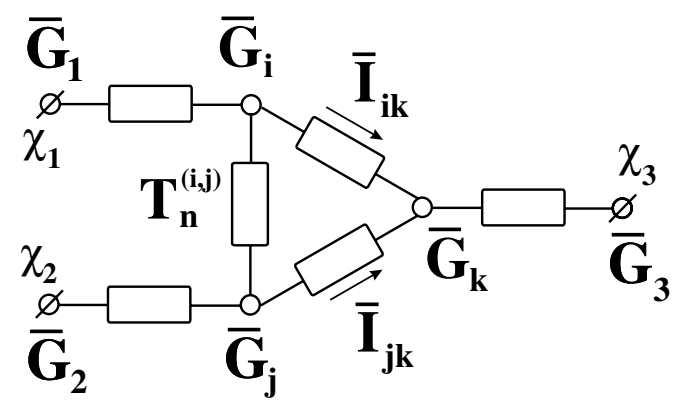

FIG. 1. The graph of the circuit theory, associated with a 3-terminal mesoscopic system. the matrix current via each connector as a function of two matrices $\bar{G}_{i, j}$ at its ends.

The connector $(i, j)$ can be quite generally characterized by a set of transmission eigenvalues $T_{n}^{(i j)}[14,15]$. The problem to solve is to express matrix current via the connector in terms of $\bar{G}_{i(j)}$. This problem shall be addressed by a more microscopic approach and was solved in [14] for the Keldysh-Nambu matrix structure of $\breve{G}$. It is good news that the derivation made in [14] does not depend on concrete matrix structure and can be used for the present problem without any modification yielding

$$
\bar{I}_{i j}=\frac{1}{2 \pi} \sum_{n} \int d E \frac{T_{n}^{(i j)}\left[\bar{G}_{i}, \bar{G}_{j}\right]}{4+T_{n}^{(i j)}\left(\left\{\bar{G}_{i}, \bar{G}_{j}\right\}-2\right)} .
$$

Each connector $(i, j)$ in the layout contributes to the total $\chi_{i}$-dependent action (2). The corresponding $S_{i j}$ contribution reads [12]

$$
\begin{aligned}
S_{i j}(\chi)= & \frac{-t_{0}}{2 \pi} \sum_{n} \int d E \\
& \times \operatorname{Tr} \ln \left[1+\frac{1}{4} T_{n}^{(i j)}\left(\left\{\bar{G}_{i}, \bar{G}_{j}\right\}-2\right)\right] .
\end{aligned}
$$

Now we are ready to present a set of circuit theory rules that enables us to evaluate the FCS for an arbitrary mesoscopic layout. (i) The layout is separated onto terminals, nodes, and connectors. (ii) The $\bar{G}_{j}$ in each terminal $j$ is fixed by relation (4) thus incorporating information about voltage, temperature, and counting field $\chi$ in each node. (iii) For each node $k$, the matrix current conservation yields a Kirchoff equation $\sum_{i} \bar{I}_{i k}=0$, where the summation is going over all connectors $(i, k)$ attached to node $k$, and $\bar{I}_{i k}$ are expressed with (6) in terms of $\bar{G}_{i(k)}$. (iv) The solution of resulting equations with condition $\bar{G}_{k}^{2}=1$ fixes $\bar{G}_{k}$ in each node. (v) The total action $S(\chi)$ is obtained by summing up the contributions $S_{i j}\left(\left\{\chi_{i}\right\}\right)$ of individual connectors; those are given by (7): $S\left(\left\{\chi_{i}\right\}\right)=\sum_{(i, j)} S_{i j}\left(\left\{\chi_{i}\right\}\right)$. (vi) The statistics of electron transfer is obtained from relation (3).

It is time to discuss the limits of applicability of the whole scheme. By virtue of the semiclassical approach, the mesoscopic fluctuations coming from interference of electrons penetrating different connectors are disregarded, so that we assume that conductivities of all connectors are much bigger than conductance quantum $e^{2} / \pi \hbar$. The same condition provides the absence of Coulomb blockade effects in the system. Besides, we have disregarded the possible processes of inelastic relaxation in the system. The latter can be eventually taken into account, since the use of the Keldysh Green functions technique allows for perturbation treatment of interaction and relaxation. However, it would considerably complicate the scheme. The point is that the inelastic scattering would mix up the $\bar{G}$ at different energies, so that one cannot solve the circuit theory equations separately at each energy.

As an illustration of the presented scheme, we consider in the rest of the Letter the FCS of the 3-terminal chaotic 
quantum dot. The system is sketched in the inset of Fig. 2. The heuristic circuit, associated with this mesoscopic system, is shown by dashed lines. It includes only three terminals, three arbitrary connectors, associated with the contacts, and the node $\{4\}$, representing the quantum dot itself. This separation is valid provided the cavity is in the quantum chaotic regime (see [16] for a definition). This regime corresponds to full isotropization of the Green function $\breve{G}\left(x, x^{\prime}, \epsilon\right)$ within the dot.

Since the normalization $\bar{G}_{k}^{2}=1$ holds for each vertex, we use the parametrization $\bar{G}_{k}=\mathbf{g}_{k} \cdot \boldsymbol{\tau}, \mathbf{g}_{k} \cdot \mathbf{g}_{k}=1$. Here $\mathbf{g}_{k}$ is a $3 \mathrm{D}$ vector, and $\boldsymbol{\tau}=\left(\bar{\tau}_{1}, \bar{\tau}_{2}, \bar{\tau}_{3}\right)$. In the absence of counting fields the Green functions in the terminals are given by a zero condition

$$
\bar{G}_{k}^{(0)}=\left(\begin{array}{cc}
1-2 f_{k} & -2 f_{k} \\
-2\left(1-f_{k}\right) & 2 f_{k}-1
\end{array}\right),
$$

where the Fermi distribution function $f_{k}(E)=\{\exp [(E-$ $\left.\left.\left.e V_{k}\right) / T_{k}\right]+1\right\}^{-1}$ accounts for the bias voltages $V_{k}$ and the temperatures $T_{k}$ in the terminals. The $\chi_{i}$ dependence of $\bar{G}_{k}(\chi)$ is then given by Eq. (4).

We see that $\bar{G}_{4}(\chi)=\mathbf{g}_{4} \cdot \boldsymbol{\tau}$ is in fact the only function to be found. For that, we proceed by applying the current conservation law, $\sum_{k=1}^{3} \bar{I}_{k, 4}=0$, inside the dot. We present the currents $\bar{I}_{k, 4}$ given by (6) in the form $\bar{I}_{k, 4}=$ $\frac{1}{2} Z_{k}\left(\mathbf{g}_{k} \cdot \mathbf{g}_{4}\right)\left[\bar{G}_{k}, \bar{G}_{4}\right]$, the scalar function $Z_{k}(x)$ incorporating the information about transmission eigenvalues in each connector $k: Z_{k}(x) \equiv \sum_{n} T_{n}^{(k, 4)} /\left[2+T_{n}^{(k, 4)}(x-1)\right]$. It can be evaluated for any particular distribution $\rho(T)$ of transmission eigenvalues in the given connector and com-

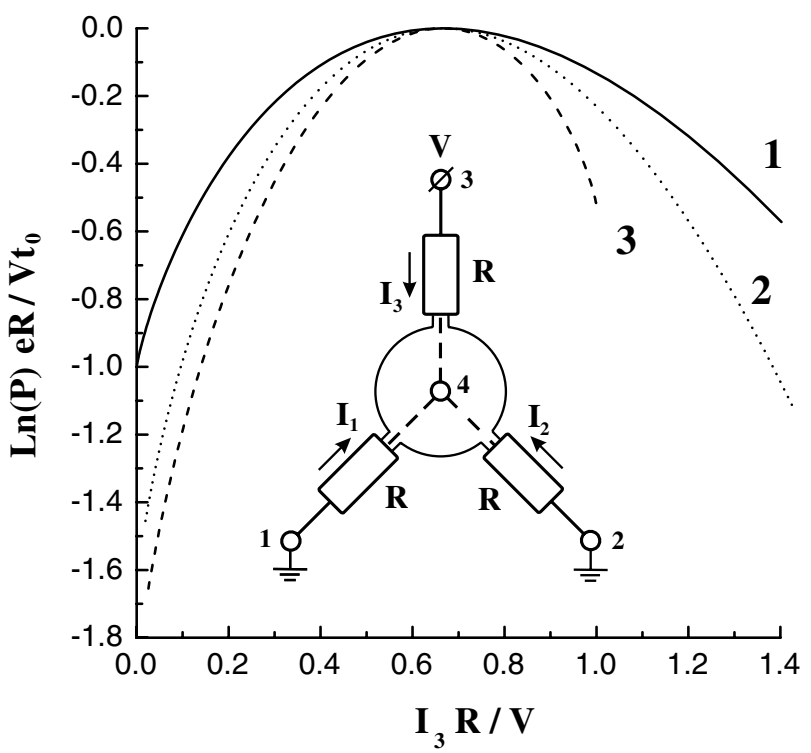

FIG. 2. The logarithm of the current probabilities in the 3-terminal chaotic quantum dot as a function of $I_{3}$, under condition $I_{1}=I_{2}$. The inset presents the system configuration. The resistances $R$ of all connectors are assumed to be equal. 1: tunnel connectors; 2: diffusive connectors; 3: ballistic connectors. pletely defines its scattering properties. For an example, if we denote $R_{0}=\pi \hbar / e^{2}$, then $R_{k}^{-1}=2 R_{0}^{-1} Z_{k}(1)$ is an inverse resistance of the connector. One can also express the Fano factor $F=\langle T(1-T)\rangle /\langle T\rangle$ as $F=1-$ $\left.2(d / d x) \log Z(x)\right|_{x=1}$. With the use of $Z_{k}(x)$ the conservation law can be efficiently rewritten as $\left[\sum_{k=1}^{3} p^{k} \bar{G}_{k}, \bar{G}_{4}\right]=$ 0 , where $p^{k}=Z_{k}\left(\mathbf{g}_{k} \cdot \mathbf{g}_{4}\right)$. The latter enables one to look for the vector $\mathbf{g}_{4}$ in the form $\mathbf{g}_{4}=M^{-1} \sum_{k=1}^{3} p^{k} \mathbf{g}_{k}$, with $M(\chi)$ being an unknown normalization constant to be determined.

The total action can be found by applying rule (v) of circuit theory and reads

$$
S(\chi)=\frac{t_{0}}{\pi} \sum_{k=1}^{3} \int d \epsilon S_{k}\left(\mathbf{g}_{k} \cdot \mathbf{g}_{4}\right)
$$

The partial contributions $S_{k}(x)$ in the above equation should be determined from the relation $\frac{\partial}{\partial x} S_{k}(x)=$ $-Z_{k}(x), S_{k}(1)=0$.

We specifically consider three particular types of connectors: tunnel $(T)$, ballistic $(B)$, and diffusive $(D)$. The corresponding contributions to action are $S_{T}(x)=$ $-\frac{1}{2}\left(R_{0} / R\right)(x-1), \quad S_{B}(x)=-\left(R_{0} / R\right) \log [(1+x) / 2]$, $S_{D}(x)=-\frac{1}{4}\left(R_{0} / R\right) \log ^{2}\left(x+\sqrt{x^{2}-1}\right)$ [10], $R$ being the resistance of the connector. For the tunnel connector $T_{n} \ll 1$ for all $n$. For the ballistic connector $N$ channels are opened $\left(T_{n} \approx 1\right.$ for $\left.n \leq N\right)$, and the others are closed. In the diffusive connector the transmission eigenvalues are distributed according to universal law $\rho(T)=R_{0} /$ $2 R T \sqrt{1-T}$.

Analytical results for FCS (9) are plausible only for the system with tunnel connectors. To assess a general situation we found $\mathbf{g}_{4}$ for given $\chi_{i}$ numerically. To find the probability distribution, we evaluated the integral (3) in the saddle point approximation, assuming $\chi_{i}$ to be complex numbers. Saddle point approximation is always valid in the low frequency limit we consider, since in this case both action $S$ and the number of transmitted particles $N_{i}=I_{i} t_{0} / e \gg 1$. Because of the current conservation law only two of three counting fields $\chi_{i}$ are independent, and one can set $\chi_{3}=0$. The relevant saddle point of the function $\Omega(\chi)=S(\chi)+i \chi_{1} I_{1} t_{0} / e+i \chi_{2} I_{2} t_{0} / e$ always corresponds to purely imaginary numbers $\left\{\chi_{1}^{*}, \chi_{2}^{*}\right\}$. The probability reads $P\left(I_{1}, I_{2}\right) \approx \exp \left[-\Omega\left(\chi^{*}\right)\right]$. Evidently, $\Omega\left(\chi^{*}\right)$ is the Legendre transform of the action, and it can be regarded as an implicit function on $I\left(\chi^{*}\right)$.

In the following we assume the shot noise regime $e V \gg$ $k T$ when the thermal fluctuations can be disregarded. The energy integration in (9) becomes trivial, since $f_{i}(\epsilon)=0$ or 1 , and it is sufficient to consider only the case $V_{1}=$ $V_{2}=0, V_{3}=V$. Any other possible setup can be reduced to the number of previous ones by appropriately subdividing a relevant energy strip. The results of these calculations are shown in Figs. 2 and 3. We see that the maximum of probability occurs at $I_{1}=I_{2}=-V / 3 R, I_{3}=2 \mathrm{~V} / 3 R$ that simply reflects the usual Kirchoff rules. The current 


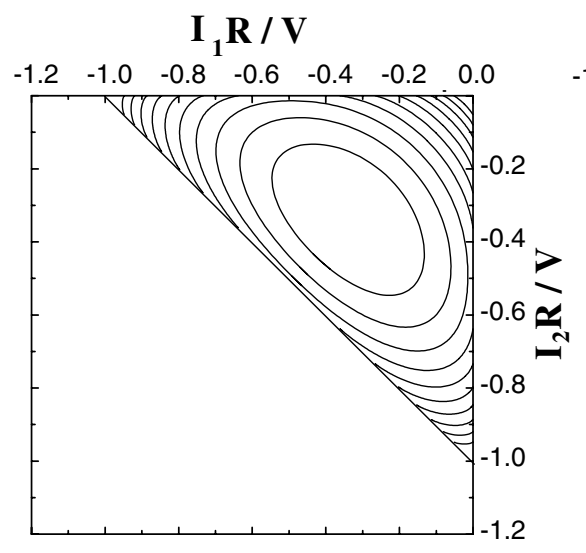

(a)

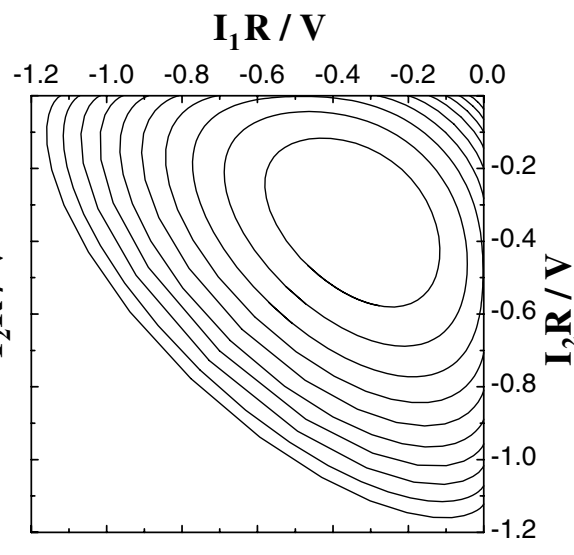

(b)

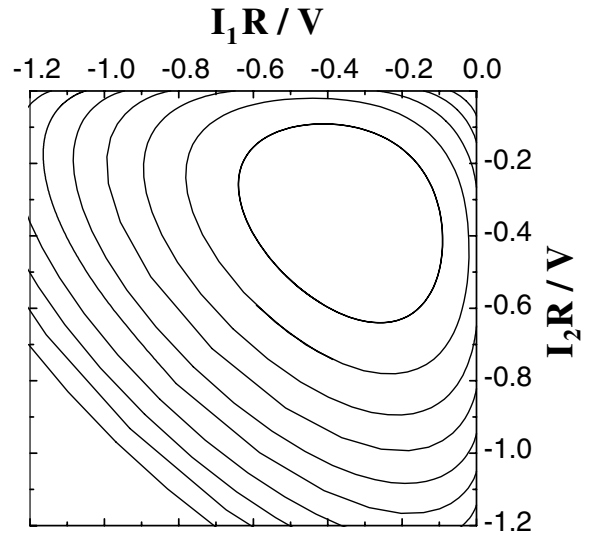

(c)

FIG. 3. The contour maps of the current distribution $\log \left[P\left(I_{1}, I_{2}\right)\right]$ in the 3-terminal chaotic quantum dot for different configurations of connectors: (a) ballistic connectors; (b) diffusive connectors; (c) tunnel connectors.

distribution $P\left(I_{1}, I_{2}\right)$ for a ballistic system is bounded. It is due to the fact that $Z_{B}(x)$ contains the finite number of open channels, contrary to the tunnel- or diffusive-type configurations, where it is not the case. From (9) we can also find a zero noise and noise correlations matrix $\tilde{S}_{i j}=e R^{-1} V F_{i j}$, $F_{11}=F_{22}=(4+3 F) / 27, F_{33}=(4+6 F) / 27, F_{12}=$ $-2 / 27, F_{13}=F_{23}=-(2+3 F) / 27$, where $F$ is a Fano factor. Since $F_{B}=0, F_{D}=1 / 3$, and $F_{T}=1$, one concludes that for a fixed average currents through connectors the Gaussian's current fluctuations will increase in the sequence ballistic $\rightarrow$ diffusive $\rightarrow$ tunnel. Figures 2 and 3 show that a similar behavior is also traced in the regime of the large current fluctuations. The essential point here is that the cross correlations always persist regardless of the concrete construction of the connectors. For the case of multilead chaotic cavities the results for shot noise in our theory coincides with Refs. [8,17].

In conclusion, we present a constructive theory for counting statistics for electron transfer in mesoscopic systems. With this theory, one can easily make theoretical predictions for all FCS, thereby facilitating experimental activities in this direction. Up to now, only the noise has been measured. In our opinion, the measurements of FCS can be easily performed with threshold detectors that produce a signal provided the current in a certain terminal exceeds the threshold value. If the threshold value exceeds the average current, the detector will be switched by relatively improbable fluctuations of the current described by FCS, its signal being proportional to the probability $P\left(I_{1}, I_{2}\right)$ of these fluctuations.

We appreciate useful discussions with G.E. W. Bauer, C. W. J. Beenakker, L. P. Kouwenhoven, and L. S. Levitov. This work is a part of the research program of the "Stichting voor Fundamenteel Onderzoek der Materie" (FOM), and we acknowledge the financial support from the "Ned- erlandse Organisatie voor Wetenschappelijk Onderzoek" (NWO).

[1] Ya. M. Blanter and M. Büttiker, Phys. Rep. 336, 1 (2000).

[2] L. Saminadayar, D. C. Glattli, Y. Jin, and B. Etienne, Phys. Rev. Lett. 79, 2526 (1997); R. de-Picciotto, M. Reznikov, M. Heiblum, V. Umansky, G. Bunin, and D. Mahalu, Nature (London) 389, 162 (1997).

[3] R. Cron, M. F. Goffman, D. Esteve, and C. Urbina, Phys. Rev. Lett. 86, 4104 (2001).

[4] A. A. Kozhevnikov, R. J. Schoelkopf, and D. E. Prober, Phys. Rev. Lett. 84, 3398 (2000).

[5] L. S. Levitov and G. B. Lesovik, JETP Lett. 58, 230 (1993); L. S. Levitov, H.-W. Lee, and G. B. Lesovik, J. Math. Phys. (N.Y.) 37, 10 (1996).

[6] M. Büttiker, Phys. Rev. B 46, 12485 (1992).

[7] R. C. Liu, B. Odom, Y. Yamamoto, and S. Tarucha, Nature (London) 391, 263 (1998).

[8] Ya. M. Blanter and E. V. Sukhorukov, Phys. Rev. Lett. 84, 1280 (2000).

[9] Ya. M. Blanter, H. Schomerus, and C. W. J. Beenakker, Physica (Amsterdam) 11E, 1 (2001).

[10] Yu. V. Nazarov, Ann. Phys. (Leipzig) 8, SI-193 (1999).

[11] J. Rammer and H. Smith, Rev. Mod. Phys. 58, 323 (1986).

[12] W. Belzig and Yu. V. Nazarov, Phys. Rev. Lett. 87, 067006 (2001); 87, 197006 (2001).

[13] A. I. Larkin and Yu. V. Ovchinninkov, Sov. Phys. JETP 41, 960 (1975); 46, 155 (1977).

[14] Yu. V. Nazarov, Superlattices Microstruct. 25, 1221 (1999).

[15] Yu. V. Nazarov, in Quantum Dynamics of Submicron Structures, edited by H. Cerdeira, B. Kramer, and G. Schoen (Kluwer, Dordrecht, The Netherlands, 1995), p. 687.

[16] O. Agam, I. Aleiner, and A. Larkin, Phys. Rev. Lett. 85, 3153 (2000).

[17] S. A. van Langen and M. Büttiker, Phys. Rev. B 56, R1680 (1997). 